\title{
Affinely Adjustable Robust Linear Complementarity Problems
}

\author{
Christian Biefel, Frauke Liers, Jan Rolfes, Martin Schmidt
}

\begin{abstract}
Linear complementarity problems are a powerful tool for modeling many practically relevant situations such as market equilibria. They also connect many sub-areas of mathematics like game theory, optimization, and matrix theory. Despite their close relation to optimization, the protection of LCPs against uncertainties - especially in the sense of robust optimization-is still in its infancy. During the last years, robust LCPs have only been studied using the notions of strict and $\Gamma$-robustness. Unfortunately, both concepts lead to the problem that the existence of robust solutions cannot be guaranteed. In this paper, we consider affinely adjustable robust LCPs. In the latter, a part of the LCP solution is allowed to adjust via a function that is affine in the uncertainty. We show that this notion of robustness allows to establish strong characterizations of solutions for the cases of uncertain matrix and vector, separately, from which existence results can be derived. Our main results are valid for the case of an uncertain LCP vector. Here, we additionally provide sufficient conditions on the LCP matrix for the uniqueness of a solution. Moreover, based on characterizations of the affinely adjustable robust solutions, we derive a mixed-integer programming formulation that allows to solve the corresponding robust counterpart. If, in addition, the certain LCP matrix is positive semidefinite, we prove polynomial-time solvability and uniqueness of robust solutions. If the LCP matrix is uncertain, characterizations of solutions are developed for every nominal matrix, i.e., these characterizations are, in particular, independent of the definiteness of the nominal matrix. Robust solutions are also shown to be unique for positive definite LCP matrix but both uniqueness and mixed-integer programming formulations still remain open problems if the nominal LCP matrix is not positive definite.
\end{abstract}

\section{INTRODUCTION}

Linear complementarity problems (LCPs) are an important tool both in mathematical theory as well as in applied mathematics. On the one hand, they serve as a bridge between mathematical fields such as optimization, game theory, and matrix theory - on the other hand, they provide one of the main modeling concepts for market equilibrium problems in energy applications like power or gas networks. For an overview of these connections, we refer to the seminal textbook [13]. Most likely, its strongest connection can be drawn to quadratic programming (QP) via the fact that the Karush-Kuhn-Tucker (KKT) conditions of many QPs can be represented as LCPs, which is also the key aspect for the applicability of LCPs in contexts such as energy markets; see, e.g., [15-17, 25].

One very active sub-area of mathematical optimization in the last decades was and is optimization under uncertainty, i.e., the study of optimization problems in which all or a certain number of parameters of the model are unknown or subject to perturbations. In order to hedge against uncertainties, two major approaches

Date: October 25, 2021.

2010 Mathematics Subject Classification. 90C33, 91B50, 91A10, 90Cxx, 90C34.

Key words and phrases. Linear Complementarity Problems, Adjustable Robustness, Robust Optimization, Existence, Uniqueness. 
have been established: stochastic optimization (see, e.g., [8, 18]) and robust optimization (see, e.g., [3, 5, 30]). While the former assumes knowledge about the distributions of the uncertain parameters and considers, e.g., the maximization of expected returns or the minimization of expected costs, the latter makes no distributional assumptions but protects against the worst-case uncertainty realization within a prescribed uncertainty set.

Although the relation between LCPs and optimization is pretty close, comparably few research papers focus on LCPs under uncertainty. Most of the related papers tackle the stochastic case and consider the minimization of the expected residual gap function of the LCP; see, e.g., [10-12, 23] and the references therein. In contrast to stochastic LCPs, the robust treatment of LCPs under uncertainty is still in its infancy. To the best of our knowledge, the first paper on robust LCPs is [31], in which the authors consider strict robustifications of LCPs. The same concept has been studied in [32, 33]. In these contributions, the authors consider strictly robust counterparts of uncertain LCPs for the case of different uncertainty sets such as box or ellipsoidal uncertainties. In particular, these papers focus on tractability of the corresponding robust counterparts. The results are applied to the case of Cournot-Bertrand equilibria in power networks in [24]; see also [9, 20] for related studies of Nash-Cournot and perfect competition equilibria in comparable settings.

The concept of strict robustness in optimization has received criticism due to the high degree of conservatism of the solutions that it may deliver. Consequently, several less conservative notions of robustness have been developed during the last twenty years; see, e.g., [6, 7, 29] for $\Gamma$-robustness, [14] for light robustness, [3, 4, 34] for adjustable robustness, or [2] for deciding robustness in a fully adjustable setting with an empty first stage. Following the idea of studying less conservative notions of robustness, the concept of $\Gamma$-robustness has been applied to LCPs in [22] for the case of $\ell_{1}$-and box-uncertainty sets and in [21] for the case of ellipsoidal uncertainties. Applications of $\Gamma$-robust LCPs in the area of power markets or traffic equilibrium problems can be found in [9, 20, 21]. To the best of our knowledge, the given and rather short list of papers on robust LCPs is complete.

Besides the study of algorithms for their solution, the most classic topic regarding LCPs is the consideration of characterizations, existence, and uniqueness of solutions. These topics closely link the field to the area of matrix classes in applied linear algebra; see again [13] and the many references therein. Unfortunately, almost all the papers on robust LCPs cited above make the observation that strong characterizations and, thus, existence of robust solutions to LCPs cannot be ensured because the requirement that a point is a complementarity solution for all realizations of uncertainty is very strong. This observation is made in [32] for strict robustness and in $[21,22]$ for $\Gamma$-robustness. As a remedy, the authors study the LCP's quadratic gap function and consider the existence and uniqueness of solutions or the tractability of problems in which the complementarity condition is not strictly demanded but in which its violation is penalized in the LCP's gap function. Thus, there is one major gap in the existing literature on robust LCPs, namely:

Is there a robustification concept that (i) allows to derive strong
characterizations of solutions of the uncertain LCP itself-instead
of the LCP's gap formulation-and that (ii) allows to establish non-
trivial robust solutions of an uncertain LCP?

To the best of our knowledge, only the concepts of strict and $\Gamma$-robustness have been studied for robust LCPs. Both do not satisfy the conditions in the question above. 
In order to cure this, it is necessary to go beyond single-stage robustness concepts - in particular, to go to two-stage robust models. Thus, in this paper, we carry over the concept of adjustable robustness to the field of LCPs under box uncertainty. The main rationale of doing so is that the split of variables into hereand-now as well as wait-and-see variables that can be adjusted to the uncertainty indeed allows to characterize robust LCP solutions and to establish non-trivial solutions. In adjustable-robust optimization, one usually first needs to specify the class of functions that can be used to adjust the wait-and-see variables in dependence of the uncertainty. The easiest functions to tackle are affine functions. Although this may be a rather restrictive choice, it is a natural modelling approach that can lead to algorithmically tractable robust counterparts [1, 26, 28]. It already gives us enough flexibility to derive strong characterizations of robust LCP solutions as well as existence results. Thus, we first focus on affine adjustability in this paper and postpone more complicated uncertainty-dependent decision rules to our future work. The class of adjustable robust LCPs is introduced in Section 2 and an illustrating example is given in Section 3. Afterward, we consider the cases of uncertain LCP vector and LCP matrix separately. Our main results are given in Section 4 for the case of uncertain LCP vector. We derive strong characterizations of robust solutions, from which an existence result is derived. The used characterizations do not require any further assumptions on the LCP matrix. This holds both for the case of full- and lower-dimensional uncertainty sets. Moreover, we illustrate exemplarily the existence of non-trivial robust LCP solutions. Uniqueness of solutions is shown for the case of positive (semi-)definite LCP matrix, in which we also obtain polynomial-time solvability. We additionally present a mixed-integer programming formulation that can be used to compute affinely adjustable robust LCP solutions by using standard solvers. Characterizations of solutions can also be derived in the case of uncertain LCP matrix; see Section 5. Here, uniqueness and tractability are shown for the case of positive definite nominal LCP matrix, whereas both remain open problems for arbitrary matrices. The paper closes with some concluding remarks and a brief discussion of possible topics of future work in Section 6 .

\section{Problem Statement}

Given a matrix $M \in \mathbb{R}^{n \times n}$ and a vector $q \in \mathbb{R}^{n}$, the linear complementarity problem $\operatorname{LCP}(q, M)$ is the problem to find a vector $z \in \mathbb{R}^{n}$ satisfying the conditions

$$
z \geq 0, \quad M z+q \geq 0, \quad z^{\top}(M z+q)=0
$$

or to show that no such vector exists. In the following, we use the standard $\perp$ notation and abbreviate (1) as

$$
0 \leq z \perp M z+q \geq 0 .
$$

In real-world applications, the parameters $M$ and $q$ may be uncertain. In order to model this, we define uncertainty sets $\mathcal{U}_{M} \subseteq \mathbb{R}^{k_{1}}$ as well as $\mathcal{U}_{q} \subseteq \mathbb{R}^{k_{2}}$ with suitable $k_{1}$ and $k_{2}$. We then consider $M(\zeta)$ and $q(u)$ with $\zeta \in \mathcal{U}_{M}$ and $u \in \mathcal{U}_{q}$. The specific definition of the uncertainty sets will be given in the corresponding sections. Since these definitions will be qualitatively different for $M$ and $q$ we choose to use a Greek letter to parameterize $M$ and a Latin letter to parameterize $q$.

We follow the robust paradigm for dealing with such uncertain parameters. In the strictly robust model, we want to find a vector $z \in \mathbb{R}^{n}$ that fulfills the conditions in (2) for every possible realization of uncertainty $(\zeta, u) \in \mathcal{U}_{M} \times \mathcal{U}_{q}$, i.e.,

$$
0 \leq z \perp M(\zeta) z+q(u) \geq 0 \quad \text { for all } \quad(\zeta, u) \in \mathcal{U}_{M} \times \mathcal{U}_{q} .
$$

We call such a vector $z$ a strictly robust solution of the uncertain LCP. This approach is discussed in $[32,33]$. The $\Gamma$-robust approach is discussed in $[21,22]$. 
The main conceptual problem with strictly as well as $\Gamma$-robust LCPs is that one usually cannot prove the existence of a solution.

The goal of this paper is to study the well established and typically less conservative approach of (affinely) adjustable robustness in the context of LCPs. For adjustable robustness, a part of the solution is allowed to adapt to a given realization of uncertainty. The task thus is to find a vector $r \in \mathbb{R}^{n}$, which can be adjusted for all uncertainties $(\zeta, u) \in \mathcal{U}_{M} \times \mathcal{U}_{q}$ by a vector $y(\zeta, u)$ so that $z(\zeta, u):=r+y(\zeta, u)$ satisfies

$$
0 \leq z(\zeta, u) \perp M(\zeta) z(\zeta, u)+q(u) \geq 0 \quad \text { for all } \quad(\zeta, u) \in \mathcal{U}_{M} \times \mathcal{U}_{q} .
$$

We call such a point $z(\zeta, u)$ an adjustable robust solution of the uncertain LCP. In many applications, further restrictions need to be imposed on the adjustable solution. For instance, one usually has to distinguish between adjustable and nonadjustable, or "here-and-now", variables. To this end, we introduce a parameter $h \in$ $\{0, \ldots, n\}$ and require that the first $h$ entries of $y(\zeta, u)$ are zero. This means that the first $h$ entries are non-adjustable here-and-now decisions.

In general, the adjustable robust approach without further assumptions on the adaptability leads to intractable problems; see, e.g., [4], where this is shown for the easiest possible case of uncertain linear programs. In this paper, we impose an assumption that is often used in adjustable robustness. Namely, we restrict ourselves to consider affinely adjustable robust solutions, i.e., we restrict the solutions to be of the form

$$
z(\zeta, u)=D_{1} \zeta+D_{2} u+r \quad \text { with } \quad D_{1} \in \mathbb{R}^{n \times k_{1}}, D_{2} \in \mathbb{R}^{n \times k_{2}}, r \in \mathbb{R}^{n} .
$$

We call an affine function $z(\zeta, u)=D_{1} \zeta+D_{2} u+r$ solving Problem (3) an affinely adjustable robust (AAR) solution of the uncertain LCP. Hence, we search for affine decision rules given by $D_{1}, D_{2}$, and $r$ that specify how to react to a given realization of uncertainty. To model $h$ here-and-now variables, we w.l.o.g. require that the first $h$ rows of $D_{1}$ and $D_{2}$ are zero.

We close this section by briefly introducing some notation that is required in the remainder of this paper. Let $A \in \mathbb{R}^{n \times n}, b \in \mathbb{R}^{n}$, and index sets $I, J \subseteq[n]:=$ $\{1, \ldots, n\}$ be given. Then, $A_{I, J} \in \mathbb{R}^{|I| \times|J|}$ denotes the submatrix of $A$ consisting of the rows indexed by $I$ and the columns indexed by $J$. Moreover, $b_{I}$ denotes the subvector with components specified by entries in $I$. If $I=J$, we also write $A_{I}$ instead of $A_{I, I}$. For $i, j \in[n]$ let $\delta_{i j}$ be the Kronecker delta, i.e., $\delta_{i j}=1$ if $i=j$ and $\delta_{i j}=0$ otherwise. Finally, the identity matrix of size $k \times k$ is denoted by $\mathbb{I}_{k}$.

\section{Illustrating Example: Adjustable Robust Energy Market EQUILIBRIUM MODELING}

In this section, we consider a stylized energy market equilibrium problem to illustrate the applicability of adjustable robustness in a practically relevant application of market modeling. To this end, we start with a simple market model based on the one given in [13] and we also follow the notation used there. First, let the production sector of our energy market model be given by the linear program

$$
\begin{aligned}
\min _{x \in \mathbb{R}^{n}} & c^{\top} x \\
\text { s.t. } & A x \geq b, \\
& B x \geq r^{*}, \\
& x \geq 0,
\end{aligned}
$$

with vectors $c \in \mathbb{R}^{n}, b \in \mathbb{R}^{m}, r^{*} \in \mathbb{R}^{k}$ as well as matrices $A \in \mathbb{R}^{m \times n}$ and $B \in \mathbb{R}^{k \times n}$. The variable vector $x$ models production levels that should be cost-minimal but that also need to satisfy certain technological constraints (4b) and demand satisfaction 
constraints $(4 \mathrm{c})$. The demand $r^{*}$ itself depends on market prices $p^{*}$, which is modeled by an affine demand function, i.e.,

$$
r^{*}=D p^{*}+d \quad \text { with } \quad D \in \mathbb{R}^{k \times k}, d \in \mathbb{R}^{k} .
$$

In many applications, one assumes that the matrix $D$ is negative semi-definite to model that demand is non-increasing in dependence of the prices. For diagonal matrices $D$ this then leads to monotonically decreasing and univariate demand functions, which is a very classic economic modeling. As usual in standard microeconomic settings, we need the additional equilibrating condition $p^{*}=\pi^{*}$ with $\pi^{*}$ being the optimal dual multiplier of the demand constraint (4c). By using this condition as well as the (necessary and sufficient) Karush-Kuhn-Tucker (KKT) conditions of Problem (4), the market equilibrium can be modeled using the LCP

$$
\begin{aligned}
& 0 \leq x \perp c-A^{\top} \lambda-B^{\top} p^{*} \geq 0, \\
& 0 \leq \lambda \perp-b+A x \geq 0, \\
& 0 \leq p^{*} \perp-D p^{*}-d+B x \geq 0,
\end{aligned}
$$

which is obtained by simplifying the KKT complementarity conditions and solving for $r^{*}$ and $\pi^{*}$. The dual multiplier of the technology constraint (4b) is denoted by $\lambda$. The corresponding LCP data is given by

$$
z=\left(\begin{array}{c}
x \\
\lambda \\
p
\end{array}\right), \quad M=\left[\begin{array}{ccc}
0 & -A^{\top} & -B^{\top} \\
A & 0 & 0 \\
B & 0 & -D
\end{array}\right], \quad q=\left(\begin{array}{c}
c \\
-b \\
-d
\end{array}\right) .
$$

If this rather general market equilibrium problem is considered as an abstract setting for an energy market, adjustable robustness in the context of LCPs shows up rather naturally. Here, the electricity demand $r^{*}$ depends on prices but also has a price-insensitive part $d$. This vector can, for instance, be estimated from historical data. However, the demand parameter $d$ is uncertain due to, e.g., unknown future weather conditions, which leads to an uncertain LCP vector $q=q(u)$ with $u$ in some properly chosen uncertainty set $\mathcal{U}_{q}$. These uncertainties in demand can usually be tackled by adjustments in production, i.e., not the "nominal" market equilibrium production is used but production is adjusted in dependence of the realization of demand uncertainty. Since, on the other hand, certain generators such as wind or solar power plants cannot be adjusted as easily as, e.g., coal power plants, this additionally leads to a rather natural split between adjustable and non-adjustable LCP variables. Note that for $D$ being negative semidefinite, the bisymmetric matrix $M$ is positive semidefinite. Thus, this practically relevant example belongs to the class of robust LCPs for which we present the strongest theoretical results in this paper - namely robust LCPs with uncertain vector $q$ and positive semi-definite matrix $M$.

Similarly, uncertainty in the coefficients of the (technological as well as demand satisfaction) constraints leads to an uncertain LCP matrix, where again some part of the solution corresponds to variables that can be adjusted, the other to those that are non-adjustable.

\section{UNCERTAINTY IN $q$}

Throughout this section we assume that the matrix $M$ is fixed and not affected by uncertainty. For a given nominal vector $\bar{q} \in \mathbb{R}^{n}$ and an uncertainty $\operatorname{set} \mathcal{U}=\mathcal{U}_{q} \subseteq \mathbb{R}^{n}$, we define $q(u):=\bar{q}+u$ for every $u \in \mathcal{U}$. The uncertain LCP (3) then reads

$$
0 \leq z(u) \perp M z(u)+q(u) \geq 0 \quad \text { for all } \quad u \in \mathcal{U} \text {. }
$$


We are interested in determining AAR solutions of (5) of the form $z(\zeta, u)=z(u)=$ $D u+r$ with $D \in \mathbb{R}^{n \times n}$ and $r \in \mathbb{R}^{n}$. To this end, we consider a box uncertainty set

$$
\mathcal{U}:=\left\{u \in \mathbb{R}^{n}:-\bar{u}_{i} \leq u_{i} \leq \bar{u}_{i}\right\}
$$

that is, w.l.o.g., centered around zero. Moreover, we split the index set $[n]$ into the set of uncertain entries

$$
U:=\left\{i \in[n]: \bar{u}_{i}>0\right\}
$$

and the set of certain entries

$$
S:=\left\{i \in[n]: \bar{u}_{i}=0\right\},
$$

i.e., $[n]=U \cup S$. For notational reasons we do not remove the columns in $D$ corresponding to $S$ but fix $D \cdot, S=0$.

Recall that we require $D_{[h], \cdot}=0$ in an AAR solution, since the first $h$ variables are non-adjustable. For a given affine function $z(u)=D u+r$, we define the sets

$$
\begin{aligned}
I & :=\left\{i \in[h]: r_{i} \neq 0\right\}, \\
J & :=\left\{i \in[n] \backslash[h]: r_{i} \neq 0\right\}, \\
K & :=\left\{i \in[n]: r_{i} \neq 0\right\}=I \cup J, \\
N & :=\left\{i \in[n]: r_{i}=0\right\}=[n] \backslash K .
\end{aligned}
$$

The assumption that the uncertainty is centered around zero immediately leads to the following key observations.

Observation 1. Let $z(u)=D u+r$ be an AAR solution of (5). Then, $r$ is a solution of the nominal $\operatorname{LCP}(\bar{q}, M)$.

Observation 2. Let $z(u)=D u+r$ be an AAR solution of (5). Since $z(u) \geq 0$ holds for all $u \in \mathcal{U}$, the inclusion

$$
\left\{i \in[n]: D_{i, U} \neq 0\right\} \subseteq J
$$

holds because, otherwise, there would exist an index $i \notin J$ and an uncertainty $u^{\prime} \in \mathcal{U}$ with $z_{i}\left(u^{\prime}\right)=D_{i, \cdot} u^{\prime}<0$.

These observations and notations will be helpful to derive the results in the following sections.

4.1. Characterization and Existence of Solutions. In this section, we show some general properties and characterizations of AAR solutions. In Lemma 2, we derive a system of equations that has to be satisfied by every AAR solution. This system of equations will be used to obtain more specific characterizations under further assumptions on the uncertainty set. Moreover, it admits an algorithmic approach to compute an AAR solution, which is addressed in Section 4.2.

First, we prove a basic lemma that reformulates the constraints in the uncertain LCP.

Lemma 1. Let $z(u)=D u+r$ and assume $D_{[h], \cdot}=0$. Then, the function $z(u)$ is an $A A R$ solution of (5) if and only if

$$
\begin{array}{rlll}
z_{K}(u) \geq 0 & \text { for all } & u \in \mathcal{U}, \\
(M z(u)+q(u))_{K}=0 & \text { for all } & u \in \mathcal{U}, \\
(M z(u)+q(u))_{N} \geq 0 & \text { for all } & u \in \mathcal{U} .
\end{array}
$$

Proof. We show that the conditions in (6) are equivalent to the uncertain LCP. By definition of $N$ and Observation 2, $z_{N}(u)=0$ holds for all $u \in \mathcal{U}$. Thus, (6a) is 
equivalent to $z(u) \geq 0$ for all $u \in \mathcal{U}$. If $z(u)$ satisfies (6b) and (6c), this implies $M z(u)+q(u) \geq 0$ for all $u \in \mathcal{U}$. Additionally, for all $u \in \mathcal{U}$ we have

$$
z(u)^{\top}(M z(u)+q(u))=z_{K}(u)^{\top}(M z(u)+q(u))_{K}+z_{N}(u)^{\top}(M z(u)+q(u))_{N}=0,
$$

where the last equality is due to $(6 \mathrm{~b})$ and $z_{N}(u)=0$. Thus, $z(u)$ satisfies (5).

It remains to show that $(6 \mathrm{~b})$ is a necessary condition. To this end, let $z(u)=$ $D u+r$ be an AAR solution. As noted before, $z_{K}(u) \geq 0$ holds for all $u \in \mathcal{U}$. Let us now assume that there is an index $i \in K$ such that there exists $u^{\prime} \in \mathcal{U}$ with $z_{i}\left(u^{\prime}\right)=0$. This implies that $u^{\prime}$ minimizes $z_{i}(u)=D_{i,} \cdot u+r_{i}$. Since $D_{i, \cdot} u+r_{i}$ is an affine function in $u$, the minimum is attained at the boundaries, i.e.,

$$
u_{j}^{\prime}= \begin{cases}\bar{u}_{j}, & \text { if } D_{i, j}<0, \\ -\bar{u}_{j}, & \text { if } D_{i, j}>0,\end{cases}
$$

for all $j$ with $D_{i, j} \neq 0$. As $r_{K}>0$, we obtain $z_{K}(u)>0$ for all $u$ contained in the relative interior relint $(\mathcal{U})$. Furthermore, the uncertain LCP conditions imply $(M z(u)+q(u))_{K}=0$ for all $u \in \operatorname{relint}(\mathcal{U})$, which immediately yields $(6 \mathrm{~b})$ since $(M z(u)+q(u))_{K}$ is an affine function in $u$ as well.

In the following, we use Condition (6b) to derive characterizations and properties of AAR solutions. In Lemma 2, we reformulate the LCP conditions and obtain a system of equations that needs to be satisfied by $D$ and $r$.

Lemma 2. The function $z(u)=D u+r$ satisfies (6b) if and only if $D$ and $r$ satisfy the system of equations

$$
\begin{aligned}
M_{K \cap S, J} D_{J, U} & =0, \\
M_{K \cap U, J} D_{J, K \cap U} & =-\mathbb{I}_{K \cap U}, \\
M_{K \cap U, J} D_{J, N \cap U} & =0, \\
M_{K} r_{K} & =-\bar{q}_{K} .
\end{aligned}
$$

Proof. Let $i \in K$. We show that $(M z(u)+q(u))_{i}=0$ holds for all $u \in \mathcal{U}$ if and only if (7) are satisfied. We have

$$
\begin{aligned}
(M z(u)+q(u))_{i} & =M_{i, \cdot} z(u)+q_{i}(u) \\
& =M_{i, \cdot} D u+M_{i, \cdot r}+\bar{q}_{i}+u_{i} \\
& =M_{i, J} D_{J,} \cdot u+M_{i, \cdot} r+\bar{q}_{i}+u_{i},
\end{aligned}
$$

where the last equality follows from $D_{i,}$. $=0$ for all $i \notin J$ by Observation 2. If $i \in K \cap S$, we have $u_{i}=0$ and thus

$$
(M z(u)+q(u))_{i}=M_{i, J} D_{J, \cdot} u+M_{i, .} r+\bar{q}_{i}=0
$$

holds for all $u \in \mathcal{U}$ if and only if

$$
M_{i, J} D_{J, U}=0 \quad \text { and } \quad M_{i, r}=-\bar{q}_{i} .
$$

If $i \in K \cap U$,

$$
(M z(u)+q(u))_{i}=M_{i, J} D_{J,} \cdot u+M_{i, \cdot} r+\bar{q}_{i}+u_{i}=0
$$

holds for all $u \in \mathcal{U}$ if and only if

$$
\begin{aligned}
M_{i, J} D_{J, j} & =-\delta_{i j} \quad \text { for all } j \in U, \\
M_{i, r} & =-\bar{q}_{i} .
\end{aligned}
$$

If the uncertainty set $\mathcal{U}$ is full-dimensional, i.e., $S=\emptyset$, the system of equations (7) is rich enough to derive a complete characterization of an AAR solution as we will show in the following. To this end, we first assume $S \subseteq[h]$, meaning that only the entries of $q(u)$ corresponding to the non-adjustable variables might be certain. 
Thus, the entries of $q(u)$ corresponding to adjustable variables are all uncertain. Under this assumption, we derive conditions that are equivalent to $(7 a)-(7 c)$ in the following lemma.

Lemma 3. Let $S \subseteq[h]$. Then, $D$ and $r$ satisfy (7a)-(7c) if and only if they satisfy the conditions

$$
\begin{aligned}
D_{J} & =-M_{J}^{-1}, \\
D_{J, i} & =0 \quad \text { for all } i \in N \cap U, \\
I \cap U & =\emptyset, \\
M_{I, J} & =0 .
\end{aligned}
$$

Proof. We first note that $S \subseteq[h]$ implies $J \subseteq U$ and thus $J \subseteq K \cap U$. Let $D$ and $r$ satisfy (7a)-(7c). We show that they satisfy (8a)-(8d). Since $J \subseteq K \cap U$, (7b) implies $M_{J} D_{J}=-\mathbb{I}_{J}$ and thus $D_{J}=-M_{J}^{-1}$, which is (8a). Furthermore, (7b) and $(7 \mathrm{c})$ imply $M_{J} D_{J, i}=0$ for all $i \in(I \cup N) \cap U$. Since $M_{J}$ has full rank, it follows $D_{J, i}=0$ for all $i \in(I \cup N) \cap U$ and thus (8b) holds as well. To show (8c), we assume that there exists an $i \in I \cap U$. However, $I \subseteq K$ and (7b) imply $M_{i, J} D_{J, i}=-1$ and thus $D_{J, i} \neq 0$, contradicting the previously proved statements. From $I \cap U=\emptyset$ and $S \subset[h]$ it follows $I=K \cap S$ and thus (7a) implies $M_{I, J} D_{J, U}=0$. In particular, $M_{I, J} D_{J}=0$ holds. Since $\operatorname{rank}\left(D_{J}\right)=|J|$, from $M_{I, J} D_{J}=0$ we obtain $M_{I, J}=0$ and thus $(8 \mathrm{~d})$.

Now, let $D$ and $r$ satisfy (8a)-(8d). By direct insertion, it is easy to verify that $D$ and $r$ satisfy $(7 \mathrm{a})-(7 \mathrm{c})$.

We can now combine Lemma 3 and Condition (7d) in Lemma 2 to obtain the desired results for the case of full-dimensional uncertainty sets, i.e., for $S=\emptyset$. The first one states that all non-adjustable variables necessarily need to have a value of zero.

Corollary 1. Let $S=\emptyset$ and suppose that $z(u)=D u+r$ is an AAR solution of (5). Then, all non-adjustable variables are zero, i.e., $I=\emptyset$ and $K=J$.

Moreover, we can use the characterizations of $D$ and $r$ from Lemma 2 and Lemma 3 to obtain a complete characterization of AAR solutions for the case of full-dimensional uncertainty sets.

Theorem 1. Let $S=\emptyset$. Then, $z(u)=D u+r$ is an $A A R$ solution of (5) if and only if $D$ and $r$ are given by

$$
\begin{aligned}
& D_{J}=-\left(M_{J}\right)^{-1}, \quad D_{J, i}=0, D_{i, \cdot}=0 \text { for all } i \notin J, \\
& r_{J}=-\left(M_{J}\right)^{-1} \bar{q}_{J}, \quad r_{i}=0 \text { for all } i \notin J
\end{aligned}
$$

and if the following conditions are fulfilled:

(a) $M_{J}$ is invertible,

(b) $-\left(M_{J}\right)^{-1} q_{J}(u) \geq 0$ for all $u \in \mathcal{U}$,

(c) $-M_{N, J}\left(M_{J}\right)^{-1} q_{J}(u)+q_{N}(u) \geq 0$ for all $u \in \mathcal{U}$.

The last theorem establishes a one-to-one correspondence between an AAR solution and the set of indices of nonzero variables $J$. Hence, to compute an AAR solution, it suffices to find a set $J$ that fulfills the conditions (a)-(c) of the theorem. Moreover, this characterization also allows to establish a finite and compact existence result for AAR solutions.

Corollary 2. Let $S=\emptyset$. For every $J \subseteq[n] \backslash[h]$, for which $M_{J}$ is invertible, we define

$$
A_{i, j}^{J}:=-\left|\left(M_{J}^{-1}\right)_{i, j} \bar{u}_{j}\right|
$$


for all $i, j \in J$ and

$$
C_{i, j}^{J}:=-\left|M_{i, \cdot}\left(M_{J}^{-1}\right) \cdot, j \bar{u}_{j}\right|
$$

for all $i \in N, j \in J$. If there exists a subset $J \subseteq[n] \backslash[h]$ such that $M_{J}$ is invertible and

$$
\begin{array}{r}
\sum_{j \in J} A_{J, j}^{J}-\left(M_{J}^{-1}\right) \bar{q}_{J} \geq 0, \\
\sum_{j \in J} C_{N, j}^{J}-\bar{u}_{N}-M_{N, J}\left(M_{J}^{-1}\right) \bar{q}_{J}+\bar{q}_{N} \geq 0,
\end{array}
$$

holds, then there exists an AAR solution.

The uniqueness, however, of an AAR solution is not given in general as shown in the following example, which also illustrates the existence of non-trivial AAR solutions.

Example 1. Consider the uncertain LCP with parameters

$$
M=\left[\begin{array}{cc}
4 & 10 \\
1 & 2
\end{array}\right], \quad \bar{q}=\left(\begin{array}{c}
-100 \\
-22
\end{array}\right), \quad \mathcal{U}=[-1,1]^{2}, \quad h=0 .
$$

There are two different AAR solutions corresponding to different index sets. For $J_{1}=\{1\}$, we obtain

$$
D=\left[\begin{array}{cc}
-\frac{1}{4} & 0 \\
0 & 0
\end{array}\right], \quad r=\left(\begin{array}{c}
25 \\
0
\end{array}\right)
$$

and for $J_{2}=\{2\}$, we have

$$
D=\left[\begin{array}{cc}
0 & 0 \\
0 & -\frac{1}{2}
\end{array}\right], \quad r=\left(\begin{array}{c}
0 \\
11
\end{array}\right) .
$$

Note that the matrix $M$ is not positive semidefinite. We later show in Section 4.3 that being positive semidefinite is a sufficient condition for an AAR solution to be unique in the case of $S=\emptyset$.

4.2. A Mixed-Integer Programming Formulation. In this section we make use of the reformulations given in Lemma 2 and state a mixed-integer feasibility problem with binary variables that can be used to compute an AAR solution of the uncertain LCP (5).

Theorem 2. Let $B \in \mathbb{R}$ be sufficiently large and consider the mixed-integer feasibility problem

$$
\begin{array}{rrr}
\text { Find } & x \in\{0,1\}^{n}, r \in \mathbb{R}^{n}, A, C, D \in \mathbb{R}^{n \times n} & \\
\text { s.t. } & B x_{i} \geq r_{i} \geq 0, & i \in[n], \\
& B\left(1-x_{i} \geq M_{i, \cdot}+\bar{q}_{i} \geq 0,\right. & \\
& D_{[h], \cdot}=0, D \cdot, S=0, & \\
& B\left(1-x_{i} \geq M_{i, \cdot} D \cdot, j \geq-B\left(1-x_{i}\right),\right. & i \in S, j \in U \\
& B\left(1-x_{j}\right)-1 \geq M_{j, \cdot} D \cdot, j \geq-B\left(1-x_{j}\right)-1, & j \in U, \\
& B\left(1-x_{i} \geq M_{i, \cdot} D \cdot, j \geq-B\left(1-x_{i}\right),\right. & i \neq j \in U, \\
& A_{i, j} \leq-D_{i, j} \bar{u}_{j}, & i \in[n], j \in U, \\
& A_{i, j} \leq D_{i, j} \bar{u}_{j}, & i \in[n], j \in U, \\
& \sum_{j \in U} A_{i, j}+r_{i} \geq 0, & i \in[n], \\
& C_{i, j} \leq-\left(M_{i, \cdot} D \cdot, j+\delta_{i j}\right) \bar{u}_{j}, & i \in[n], j \in U, \\
& C_{i, j} \leq\left(M_{i, \cdot} D \cdot, j+\delta_{i j}\right) \bar{u}_{j}, & i \in[n], j \in U,
\end{array}
$$




$$
\sum_{j \in U} C_{i, j}+M_{i, r}+\bar{q}_{i} \geq 0
$$

If (9) is feasible, it returns an AAR solution of the form $z(u)=D u+r$ to (5). If it is infeasible, then no AAR solution exists.

Proof. It suffices to show that every solution of (9) corresponds to an AAR solution and vice versa. First, let $(x, r, A, C, D)$ be a solution of (9). Note that $D$ fulfills the basic requirements $D_{[h], \cdot}=0$ and $D \cdot, S=0$ by $(9 \mathrm{~d})$. We now show that $z(u)=$ $D u+r$ is an AAR solution. The inequality

$$
\sum_{j \in U} A_{i, j} \leq \min _{u \in \mathcal{U}}\left\{D_{i,}, u\right\}
$$

holds for all $i \in[n]$ by (9h) and (9i). It follows

$$
\min _{u \in \mathcal{U}}\left\{z_{i}(u)\right\} \geq \sum_{j \in U} A_{i, j}+r_{i} \geq 0
$$

for all $i \in[n]$, where the last inequality follows from $(9 \mathrm{j})$. This implies $z(u) \geq 0$ for all $u \in \mathcal{U}$. In particular, since $r_{N}=0$, we also obtain $D_{N, U}=0$ and hence $z_{N}(u)=0$ for all $u \in \mathcal{U}$. Due to (9b), we have $x_{i}=1$ if $i \in K$. Thus, (9c) implies (7d) and $(9 \mathrm{e})-(9 \mathrm{~g})$ imply the conditions $(7 \mathrm{a})-(7 \mathrm{c})$. Hence, $(6 \mathrm{~b})$ holds due to Lemma 2, i.e., $(M z(u)+q(u))_{K}=0$ for all $u \in \mathcal{U}$. From $z_{N}(u)=0$ and $(M z(u)+q(u))_{K}=0$ for all $u \in \mathcal{U}$ it immediately follows $z(u)^{\top}(M z(u)+q(u))=0$ for all $u \in \mathcal{U}$.

It remains to show that $(M z(u)+q(u))_{N} \geq 0$ holds for all $u \in \mathcal{U}$. The inequalities (9k) and (9l) imply

$$
C_{i, j} \leq \min _{u \in \mathcal{U}}\left\{M_{i, .} D_{\cdot, j} u_{j}+\delta_{i j} u_{j}\right\}
$$

for all $i \in[n], j \in U$. Hence, we obtain

$$
\sum_{j \in U} C_{i, j} \leq \min _{u \in \mathcal{U}}\left\{M_{i, .} D u+u_{i}\right\}
$$

for all $i \in N \subseteq[n]$. It follows

$$
\min _{u \in \mathcal{U}}\left\{(M z(u)+q(u))_{i}\right\} \geq \sum_{j \in U} C_{i, j}+M_{i, r}+\bar{q}_{i} \geq 0
$$

for all $i \in N$, where the last inequality follows from $(9 \mathrm{~m})$. Thus, $(M z(u)+q(u))_{N} \geq$ 0 holds for all $u \in \mathcal{U}$.

Now, let $z(u)=D u+r$ be an AAR solution of (5). Next, we construct $x, A$, and $C$ such that $(x, r, A, C, D)$ is a solution of (9). For all $i \in K$, we set $x_{i}=1$ and for all $i \in N$ we set $x_{i}=0$. Since $r$ is a nominal solution, the constraints (9b) and (9c) are satisfied for sufficiently large $B$. Since $D$ fulfills the basic requirements $D_{h, \cdot}=0$ and $D_{\cdot, S}=0$, Condition (9d) is satisfied. Furthermore, $D$ is a solution of the equations (7a)-(7c) in Lemma 2 and, thus, $D$ satisfies (9e)-(9g) for sufficiently large $B$. Next, we define $A_{i, j}:=-\left|D_{i, j} \bar{u}_{j}\right|$ for all $i, j \in[n]$. Then, (9h) and (9i) are satisfied, implying

$$
\sum_{j \in U} A_{i, j}+r_{i}=\min _{u \in \mathcal{U}}\left\{z_{i}(u)\right\} \geq 0
$$

for all $i \in[n]$. Hence, $(9 \mathrm{j})$ is satisfied. Lastly, we define

$$
C_{i, j}:=-\left|\left(M_{i, \cdot} D \cdot, j+\delta_{i j}\right) \bar{u}_{j}\right|
$$

for all $i, j \in[n]$. Then, (9k) and (9l) are satisfied, implying

$$
\sum_{j \in U} C_{i, j}+M_{i, r}+\bar{q}_{i}=\min _{u \in \mathcal{U}}\left\{(M z(u)+q(u))_{i}\right\} \geq 0
$$

for all $i \in[n]$. Hence, $(9 \mathrm{~m})$ is satisfied. 
Remark 1. One crucial aspect regarding the correctness of the binary feasibility problem in Theorem 2 is that the constant $B$ needs to be sufficiently large. For general LCPs, it can be computationally expensive to compute this constant; see, e.g., [27]. However, for specific instances, problem-specific structure can often be exploited to obtain such constants; see, e.g., [19], where similar constants are derived by using the specific structure of a market equilibrium problem that can also be modeled as a complementarity problem.

4.3. Positive Semidefinite $M$. In the remainder of this section, we assume that the matrix $M$ is positive semidefinite. In this case, we attain polynomial-time solvability and uniqueness results under further assumptions on the uncertainty set $\mathcal{U}$. First, we review the following well established theorem on linear complementarity problems.

Lemma 4 (Theorem 3.1.7 (a), (c) in [13]). Let $M \in \mathbb{R}^{n \times n}$ be positive semidefinite and let $q \in \mathbb{R}^{n}$ be chosen arbitrarily. Then, the following assertions hold.

(a) If $z^{1}$ and $z^{2}$ are two solutions of the $\operatorname{LCP}(q, M)$, then

$$
\left(z^{1}\right)^{\top}\left(q+M z^{2}\right)=\left(z^{2}\right)^{\top}\left(q+M z^{1}\right)=0 .
$$

(b) If the $\operatorname{LCP}(q, M)$ has a solution, then the set $S O L(q, M)$ of solutions is polyhedral and given by

$$
\begin{gathered}
S O L(q, M)=\left\{z \in \mathbb{R}_{\geq 0}^{n}: q+M z \geq 0, q^{\top}(z-\bar{z})=0,\right. \\
\left.\left(M+M^{\top}\right)(z-\bar{z})=0\right\},
\end{gathered}
$$

where $\bar{z}$ is an arbitrary solution.

For what follows, we define

$$
P:=\left\{j \in[n]: \exists z \in \operatorname{SOL}(\bar{q}, M): z_{j}>0\right\}, \quad L:=[n] \backslash P .
$$

For the following results, we need to know the index set $P$ explicitly. Note that $\operatorname{SOL}(\bar{q}, M)$ can be explicitly stated via Part (b) of the previous lemma since the special solution $\bar{z}$ can be computed by solving a single convex quadratic program. The set $P$ can then be obtained by solving $n$ linear programs in which $z_{j}, j \in[n]$, is maximized over the polyhedral feasible set $\operatorname{SOL}(\bar{q}, M)$ and by checking afterward, whether the solution is strictly positive. Thus, $P$ can be computed in polynomial time.

We now use Lemma 4 to strengthen Lemma 2.

Lemma 5. Let $M$ be positive semidefinite. If $z(u)=D u+r$ is an $A A R$ solution of (5), the system of equations

$$
\begin{aligned}
M_{P \cap S, P} D_{P, U} & =0, \\
M_{P \cap U, P} D_{P, P \cap U} & =-\mathbb{I}_{P \cap U}, \\
M_{P \cap U, P} D_{P, L \cap U} & =0 .
\end{aligned}
$$

is satisfied.

Proof. From Observation 1 we know that $r$ is a nominal solution. Thus, due to Lemma 4 (a), $M_{P, r}+\bar{q}_{P}=0$ holds. Since $z(u)=D u+r$ is an AAR solution, we know

$$
M z(u)+q(u)=M D u+M r+\bar{q}+u \geq 0
$$

for all $u \in \mathcal{U}$. In particular, we have

$$
(M D u+M r+\bar{q}+u)_{P}=M_{P, \cdot} D u+M_{P, \cdot}+\bar{q}_{P}+u_{P}=M_{P, \cdot} D u+u_{P} \geq 0
$$


for all $u \in \mathcal{U}$. Since we set $D_{\cdot, S}=0$, we have $D_{P, \cdot} u=D_{P, U} u_{U}$ and from Observation 2 it follows $M_{P, \cdot} D=M_{P} D_{P, .}$ Hence, the inequality

$$
M_{P, P} D_{P, U} u_{U}+u_{P} \geq 0
$$

holds for all $u \in \mathcal{U}$.

For $i \in P \cap S$, we have $u_{i}=0$ and, thus, $M_{i, P} D_{P, U} u_{U} \geq 0$ holds for all $u \in \mathcal{U}$. This implies $M_{i, P} D_{P, U}=0$ as otherwise there would exist an element $u^{\prime} \in \mathcal{U}$ from the uncertainty set defined by $u_{U}^{\prime}=-\lambda\left(M_{i, P} D_{P, U}\right)^{\top}$ for some $\lambda>0$ and $u_{S}^{\prime}=0$ so that $M_{i, P} D_{P, U} u_{U}^{\prime}=-\lambda\left\|M_{i, P} D_{P, U}\right\|_{2}<0$. Thus, (10a) holds.

Next, for $i \in P \cap U$ we have $M_{i, P} D_{P, U} u_{U}+u_{i} \geq 0$ for all $u \in \mathcal{U}$. For the same reasons as in the previous case, this implies $M_{i, P} D_{P, U} u_{U}=-u_{i}$, as otherwise we could again construct an uncertainty $u^{\prime}$ in the box uncertainty set $\mathcal{U}$ so that $M_{i, P} D_{P, U} u_{U}^{\prime}+u_{i}^{\prime}<0$. We obtain (10b) and (10c).

We now combine Lemma 4 and 5 to obtain a linear feasibility problem that can be used to solve the uncertain LCP with positive semidefinite $M$. Thus, in this case, there is no need to solve the mixed-integer feasibility problem from Theorem 2.

Theorem 3. Let $M$ be positive semidefinite and suppose further that $\bar{z}$ is a solution of the nominal $\operatorname{LCP}(\bar{q}, M)$. Consider the linear feasibility problem

$$
\begin{array}{ll}
\text { Find } & r \in \mathbb{R}^{n}, A, C, D \in \mathbb{R}^{n \times n} \\
\text { s.t. } \quad & r \geq 0, \bar{q}+M r \geq 0, \\
& \bar{q}^{\top}(r-\bar{z})=0, \\
& \left(M+M^{\top}\right)(r-\bar{z})=0, \\
& D_{L, \cdot}=0, D_{[h], \cdot}=0, \quad D \cdot, S=0, \\
& {\left[M_{P \cap S, P}\right]\left[D_{P, P \cap U} \quad D_{P, L \cap U}\right]=\left[\begin{array}{cc}
0 & 0 \\
-\mathbb{I}_{P \cap U} & 0
\end{array}\right],} \\
& \left.M_{P \cap U, P}\right], \\
& A_{i, j} \leq-D_{i, j} \bar{u}_{j}, \quad i \in P, j \in U, \\
& A_{i, j} \leq D_{i, j} \bar{u}_{j}, \quad i \in P, j \in U, \\
& \sum_{j \in U} A_{i, j}+r_{i} \geq 0, \quad i \in P, \\
& C_{i, j} \leq-\left(M_{i, \cdot} D \cdot, j+\delta_{i j}\right) \bar{u}_{j}, \quad i \in L, j \in U, \\
& C_{i, j} \leq\left(M_{i, \cdot} D \cdot, j+\delta_{i j}\right) \bar{u}_{j}, \quad i \in L, j \in U, \\
& \sum_{j \in U} C_{i, j}+M_{i, \cdot}+\bar{q}_{i} \geq 0, \quad i \in L .
\end{array}
$$

Every feasible point of (11) corresponds to an $A A R$ solution of the form $z(u)=$ $D u+r$. If (11) is infeasible, then no AAR solution exists.

As parts of the proof of Theorem 3 are similar to that of Theorem 2, we keep the following proof rather short.

Proof. Let $(r, A, C, D)$ be a solution of (11). We show, that $z(u)=D u+r$ is an AAR solution. First, we note that $D$ satisfies the basic requirements $D_{[h],}$. $=0$ and $D,_{, S}=0$ by (11d). Since $r$ satisfies (11a)-(11c), it is a solution of the nominal $\operatorname{LCP}(\bar{q}, M)$ by Lemma 4 (b). Therefore, we obtain $r_{L}=0$ by the definition of $P$ and $L, D_{L, \cdot}=0$ by $(11 \mathrm{~d})$ and thus $z_{L}(u)=0$ holds for all $u \in \mathcal{U}$. Furthermore, we know $(M r+\bar{q})_{P}=0$ due to Lemma 4 (a). From (11e) it follows $(M D u)_{P}=-u_{P}$ and thus

$$
(M z(u)+q(u))_{P}=(M D u)_{P}+u_{P}+(M r+\bar{q})_{P}=0
$$


holds for all $u \in \mathcal{U}$. From $z_{L}(u)=0$ and $(M z(u)+q(u))_{P}=0$ for all $u \in \mathcal{U}$ it follows $z(u)^{\top}(M z(u)+q(u))=0$ for all $u \in \mathcal{U}$.

It remains to show that $z_{P}(u) \geq 0$ and $(M z(u)+q(u))_{L} \geq 0$ holds for all $u \in \mathcal{U}$. The constraints (11f)-(11h) imply $z_{P}(u) \geq 0$ for all $u \in \mathcal{U}$ and the constraints (11i)-(11k) imply $(M z(u)+q(u))_{L} \geq 0$ for all $u \in \mathcal{U}$ for the same reasons as in the proof of Theorem 2.

Now, let $z(u)=D u+r$ be an AAR solution. We construct $A$ and $C$ such that $(r, A, C, D)$ is a solution of (11). We know that $r$ is a nominal solution and, thus, (11a)-(11c) are satisfied by Lemma 4(b). By definition of $P$ and $L$, we have $r_{L}=0$ and thus $D_{L, U}=0$ due to Observation 2. The requirements $D_{h, \cdot}=0$ and $D_{\cdot, S}=0$ hold by definition. Hence, (11d) is satisfied. The constraint (11e) holds due to Lemma 5. For all $i, j \in[n]$, we now define

$$
\begin{aligned}
A_{i, j} & :=-\left|D_{i, j} \bar{u}_{j}\right|, \\
C_{i, j} & :=-\left|\left(M_{i, \cdot} D_{\cdot, j}+\delta_{i j}\right) \bar{u}_{j}\right| .
\end{aligned}
$$

Then, (11f)-(11k) are satisfied for the same reasons as in the proof of Theorem 2.

If the matrix $M$ is positive semidefinite, the nominal LCP can be solved by solving a convex quadratic program. Therefore, a solution $\bar{z}$ for the nominal LCP, which we need as a precondition in Theorem 3, can be computed in polynomial time. Since the linear feasibility problem (11) can be solved in polynomial time as well, we obtain the following complexity result.

Corollary 3. Let $M$ be positive semidefinite. Then one can find an AAR solution of (5) or correctly state that there is no AAR solution in polynomial time.

We now use Lemma 5 to obtain uniqueness results under additional assumptions on the uncertainty set. As in the general case in Section 4.1, we first consider the case $S \subseteq[h]$.

Lemma 6. Let $M$ be positive semidefinite and $S \subseteq[h]$. If $z(u)=D u+r$ is an $A A R$ solution of (5), the matrix $D$ is uniquely determined by $D_{P \cap U}=-\left(M_{P \cap U}\right)^{-1}$ and $D_{i, j}=0$ for all $i, j \notin P \cap U$.

Proof. From Lemma 5 we know

$$
\left[\begin{array}{ll}
-\mathbb{I}_{P \cap U} & 0
\end{array}\right]=M_{P \cap U, P}\left[\begin{array}{ll}
D_{P, P \cap U} & D_{P, L \cap U}
\end{array}\right] .
$$

Since $D_{[h], \cdot}=0$ and $S \subseteq[h]$ holds, we have $D_{P \cap S, \cdot}=0$, which implies

$$
\left[\begin{array}{ll}
-\mathbb{I}_{P \cap U} & 0
\end{array}\right]=M_{P \cap U, P}\left[\begin{array}{ll}
D_{P, P \cap U} & D_{P, L \cap U}
\end{array}\right]=M_{P \cap U}\left[\begin{array}{ll}
D_{P \cap U} & D_{P \cap U, L \cap U}
\end{array}\right] .
$$

Thus, the equation $M_{P \cap U} D_{P \cap U}=-\mathbb{I}_{P \cap U}$ implies $D_{P \cap U}=-\left(M_{P \cap U}\right)^{-1}$. Furthermore, since $M_{P \cap U}$ is invertible and $M_{P \cap U} D_{P \cap U, L \cap U}=0$ holds, it follows $D_{P \cap U, L \cap U}=0$. As $D_{i, .}=0$ for all $i \notin P$ due to Observation 2, this finishes the proof.

The previous lemma asserts the uniqueness of the matrix $D$. If we now assume that all entries of $q(u)$ are uncertain, i.e., $S=\emptyset$, Lemma 6 leads to uniqueness of the entire AAR solution.

Theorem 4. Let $M$ be positive semidefinite and $S=\emptyset$.

(a) If there are multiple solutions to the nominal $L C P(\bar{q}, M)$, there is no $A A R$ solution.

(b) If there exists an $A A R$ solution, it is unique.

Proof. We first note that $P \cap U=P$ holds since $S=\emptyset$. Any solution $r$ to the nominal $\operatorname{LCP}(\bar{q}, M)$ satisfies $M_{P} r_{P}=-\bar{q}_{P}$ due to Lemma $4($ a) and the definition of $P$. If there are multiple solutions, $M_{P}$ cannot be invertible and, thus, there 
cannot exist an AAR solution according to Lemma 6. Hence, if there is an AAR solution $z(u)=D u+r, r$ is unique due to the previous argument and $D$ is unique due to Lemma 6.

We close this section with some remarks on the connection between our results and the classical LCP theory as well as on the limits of affine adjustability. If the matrix $M$ is positive semidefinite, the nominal LCP can be solved by solving a convex QP, which can be done in polynomial time. This is also the underlying reason for our complexity result Corollary 3. As for nominal LCPs, uniqueness of solutions cannot be guaranteed in the case of an arbitrary matrix $M$. Under the assumption that $M$ is a $P$ matrix, i.e., all principal minors of $M$ are positive, the uniqueness of the solution of the nominal LCP is guaranteed for every $q$; see, e.g., [13, Chapter 3]. This statement directly carries over to uncertain LCPs with general uncertainty sets. If the solution $z(u)$ for every realization of the uncertainties $u \in \mathcal{U}$ is unique, an AAR solution is unique as well. However, Theorem 4 states that, in the case of full-dimensional uncertainty sets, we only need positive semidefiniteness of the matrix $M$ to guarantee the uniqueness of an AAR solution, which is a less strong condition than $M$ being a $P$ matrix.

Note that we illustrated the existence of non-trivial solutions, see Example 1, and stated conditions for the existence of a solution in Corollary 2. However, let us also note that there exist uncertain LCPs that have an adjustable but not an affinely adjustable robust solution as the following example shows.

Example 2. Consider the uncertain LCP given by

$$
M=\left[\begin{array}{cc}
1 & \frac{1}{2} \\
\frac{1}{2} & 1
\end{array}\right] \succ 0, \quad \bar{q}=\left(\begin{array}{l}
-5 \\
-3
\end{array}\right), \quad \mathcal{U}=[-1,1]^{2}, \quad h=0 .
$$

Since all principal minors of $M$ are positive, $M$ is a $P$ matrix. Hence, for any realization $u^{\prime} \in \mathcal{U}$, there exists a solution of the nominal $\operatorname{LCP}\left(q\left(u^{\prime}\right), M\right)$. Therefore, a fully adjustable solution would map every realization to its respective unique solution. However, the uncertain LCP does not have an AAR solution, which can be verified by applying Theorem 1 .

Solving the uncertain LCP with other decision rules than affine ones is left for future research.

\section{UNCERTAINTY IN $M$}

In this section, we assume that the vector $q$ is certain and consider uncertainty only in the matrix $M$. In particular, we are given matrices $M^{0}, M^{1}, \ldots, M^{k} \in \mathbb{R}^{n \times n}$ as well as $\mathcal{U}=\mathcal{U}_{M}=[-1,1]^{k}$ and define

$$
M(\zeta):=M^{0}+\sum_{i=1}^{k} \zeta_{i} M^{i}
$$

The uncertain LCP (3) then reads

$$
0 \leq z(\zeta) \perp M(\zeta) z(\zeta)+q \geq 0 \quad \text { for all } \quad \zeta \in \mathcal{U} .
$$

For this problem, we are interested in computing an AAR solution of the form $z(\zeta)=D \zeta+r$ with $D \in \mathbb{R}^{n \times k}$ and $r \in \mathbb{R}^{n}$. As before, we assume that the first $h$ rows of $D$ are zero for some fixed $h$ to distinguish between adjustable and nonadjustable variables. However, the results presented in this section are independent of the specific choice of $h$.

Remark 2. We can interpret $M^{0}$ as the nominal matrix that is perturbed by the matrices $M^{1}, \ldots, M^{k}$. This definition of a matrix uncertainty set is considered in [33] for the first time in the context of LCPs and is also used in [22]. 
For an AAR solution $z(\zeta)=D \zeta+r$ we define the sets

$$
J:=\left\{j \in[n]: r_{j}>0\right\}, \quad N:=[n] \backslash J .
$$

As in Observation 2 for the case of uncertain $q$, we have $\left\{j \in[n]: D_{j,} \neq 0\right\} \subseteq J$ and, thus, $D_{N, \cdot}=0$. Analogously to the proof of Lemma 1, we have $z_{J}(\zeta)>0$ for all $\zeta \in \operatorname{int}(\mathcal{U})$.

We now prove necessary conditions that every AAR solution satisfies.

Theorem 5. Let $z(\zeta)=D \zeta+r$ be an AAR solution for (12). Then,

$$
\begin{array}{rlll}
M_{J}^{0} r_{J}+q_{J} & =0, & & \\
M_{J}^{i} r_{J}+M_{J}^{0} D_{J, i}=0 & \text { for all } & i \in[k], \\
M_{J}^{i} D_{J, i}=0 & \text { for all } & i \in[k], \\
M_{J}^{i} D_{J, j}+M_{J}^{j} D_{J, i}=0 & \text { for all } & i, j \in[k], i \neq j
\end{array}
$$

holds.

Proof. Since $0 \in \mathcal{U}$, the vector $z(0)=r$ is a solution of the nominal $\operatorname{LCP}\left(q, M^{0}\right)$ and thus (13a) holds. For $i \in[k]$, we define

$$
\mathcal{U}_{i}:=\left\{\zeta \in \mathcal{U}: \zeta_{i} \in(-1,1), \zeta_{j}=0 \text { for all } j \neq i\right\} \subseteq \operatorname{int}(\mathcal{U}) .
$$

We have $z_{J}(\zeta)>0$ for all $\zeta \in \mathcal{U}_{i}$ and thus $(M(\zeta) z(\zeta)+q)_{J}=0$ holds for all $\zeta \in \mathcal{U}_{i}$. We obtain

$$
\begin{aligned}
0 & =\left(M_{J}^{0}+\zeta_{i} M_{J}^{i}\right)\left(r_{J}+\zeta_{i} D_{J, i}\right)+q_{J} \\
& =M_{J}^{0} r_{J}+\zeta_{i}\left(M_{J}^{i} r_{J}+M_{J}^{0} D_{J, i}\right)+\zeta_{i}^{2} M_{J}^{i} D_{J, i}+q_{J}
\end{aligned}
$$

for all $\zeta_{i} \in(-1,1)$. Hence, the conditions (13b) and (13c) follow.

Now, for $i, j \in[k]$ with $i \neq j$, we define

$$
\mathcal{U}_{i, j}:=\left\{\zeta \in \mathcal{U}: \zeta_{i}, \zeta_{j} \in(-1,1), \zeta_{p}=0 \text { for all } p \notin\{i, j\}\right\} \subseteq \operatorname{int}(\mathcal{U}) .
$$

As before, $z_{J}(\zeta)>0$ holds for all $\zeta \in \mathcal{U}_{i, j}$ and thus

$$
\begin{aligned}
0= & (M(\zeta) z(\zeta)+q)_{J} \\
= & M(\zeta)_{J}(D \zeta+r)_{J}+q_{J} \\
= & M_{J}^{0}\left(\zeta_{i} D_{J, i}+\zeta_{j} D_{J, j}+r_{J}\right)+\zeta_{i} M_{J}^{i}\left(\zeta_{i} D_{J, i}+\zeta_{j} D_{J, j}+r_{J}\right) \\
& +\zeta_{j} M_{J}^{j}\left(\zeta_{i} D_{J, i}+\zeta_{j} D_{J, j}+r_{J}\right)+q_{J} \\
= & \left(M_{J}^{0} r_{J}+q_{J}\right)+\zeta_{i}\left(M_{J}^{0} D_{J, i}+M_{J}^{i} r_{J}\right)+\zeta_{j}\left(M_{J}^{0} D_{J, j}+M_{J}^{j} r_{J}\right) \\
& +\zeta_{i}^{2} M_{J}^{i} D_{J, i}+\zeta_{j}^{2} M_{J}^{j} D_{J, j}+\zeta_{i} \zeta_{j}\left(M_{J}^{i} D_{J, j}+M_{J}^{j} D_{J, i}\right)=(*)
\end{aligned}
$$

for all $\zeta \in \mathcal{U}_{i, j}$. The first term is zero due to (13a). Applying (13b) and (13c), all other terms except for the last one are zero as well. It follows

$$
0=(*)=\zeta_{i} \zeta_{j}\left(M_{J}^{i} D_{J, j}+M_{J}^{j} D_{J, i}\right)
$$

for all $\zeta \in \mathcal{U}_{i, j}$ and thus (13d) holds.

Since the systems of equations of the last theorem might allow for multiple solutions, they are not sufficient to fully characterize an AAR solution. However, under the additional assumption that $M_{J}^{0}$ is invertible, it is possible to derive a complete characterization. For example, this assumption is satisfied if $M^{0}$ is positive definite as in this case every submatrix $M_{I}^{0}, I \subseteq[n]$, is invertible.

We first introduce some notation and subsequently present the complete characterization in Corollary 4. To this end, let $M_{I}^{0}$ be invertible for a subset $I \subseteq[n]$. Then, we define

$$
\tilde{M}^{I, i}:=\left(M_{I}^{0}\right)^{-1} M_{I}^{i}\left(M_{I}^{0}\right)^{-1} .
$$


Corollary 4. Let $z(\zeta)=D \zeta+r$ be an $A A R$ solution for (12). If $M_{J}^{0}$ is invertible, then $D$ and $r$ are given by

$$
D_{J, i}=\tilde{M}^{J, i} q_{J}, i \in[k], \quad r_{J}=-\left(M_{J}^{0}\right)^{-1} q_{J}, \quad D_{N, \cdot}=0, \quad r_{N}=0 .
$$

Proof. Since $M_{J}^{0}$ is invertible, (13a) is equivalent to $r_{J}=-\left(M_{J}^{0}\right)^{-1} q_{J}$. By using this equation for $q_{J},(13 \mathrm{~b})$ can be equivalently reformulated as $M_{J}^{0} D_{J, i}=M_{J}^{i}\left(M_{J}^{0}\right)^{-1} q_{J}$ for all $i \in[k]$. Thus, for all $i \in[k]$ we obtain

$$
D_{J, i}=\left(M_{J}^{0}\right)^{-1} M_{J}^{i}\left(M_{J}^{0}\right)^{-1} q_{J}=\tilde{M}^{J, i} q_{J} .
$$

In the next example, we illustrate that indeed solutions characterized by this corollary exist.

Example 3. Let

$$
M(\zeta)=\left[\begin{array}{ll}
4 & 1 \\
0 & 4
\end{array}\right]+\zeta\left[\begin{array}{ll}
0 & 1 \\
0 & 0
\end{array}\right], \quad q=\left(\begin{array}{c}
-8 \\
-16
\end{array}\right), \quad h=0 .
$$

As $M^{0}$ is invertible, we consider the set $J=[n]$. It follows

$$
\left(M^{0}\right)^{-1}=\frac{1}{16}\left[\begin{array}{cc}
4 & -1 \\
0 & 4
\end{array}\right] \text { and } \quad \tilde{M}^{J, 1}=\left(M^{0}\right)^{-1} M^{1}\left(M^{0}\right)^{-1}=\frac{1}{16}\left[\begin{array}{ll}
0 & 1 \\
0 & 0
\end{array}\right] \text {. }
$$

Using Corollary 4, we obtain

$$
r=-\left(M^{0}\right)^{-1} q=\left(\begin{array}{l}
1 \\
4
\end{array}\right), \quad D=\tilde{M}^{J, 1} q=\left(\begin{array}{c}
-1 \\
0
\end{array}\right) .
$$

It is easy to verify that $z(\zeta)=(1-\zeta, 4)^{\top}$ is an AAR solution.

For what follows, let $z(\zeta)=D \zeta+r$ be an AAR solution and suppose that $M_{J}^{0}$ is invertible. The conditions (13c) and (13d) can be reformulated similarly as in the proof of Corollary 4 by using the characterizations of $r$ and $D$. We obtain that (13c) is equivalent to

$$
M_{J}^{i} \tilde{M}^{J, i} q_{J}=0 \quad \text { for all } \quad i \in[k] .
$$

Expression (13d) is equivalent to

$$
\left(M_{J}^{i} \tilde{M}^{J, j}+M_{J}^{j} \tilde{M}^{J, i}\right) q_{J}=0 \quad \text { for all } \quad i, j \in[k], i \neq j .
$$

We combine these conditions and obtain

$$
q_{J} \in \bigcap_{i, j \in[k]} \operatorname{ker}\left(M_{J}^{i} \tilde{M}^{J, j}+M_{J}^{j} \tilde{M}^{J, i}\right) .
$$

In the following, we derive a reformulation of the uncertain LCP conditions in (12) such that they only depend on the LCP parameters $M$ and $q$. To this end, we use Corollary 4. The equation

$$
D_{J,} \zeta=\sum_{i=1}^{k} \zeta_{i} \tilde{M}^{J, i} q_{J}
$$

holds for all $\zeta \in \mathcal{U}$. Thus, the requirement that $z_{J}(\zeta) \geq 0$ for all $\zeta \in \mathcal{U}$ is equivalent to

$$
\left(\sum_{i \in[k]} \zeta_{i} \tilde{M}^{J, i}-\left(M_{J}^{0}\right)^{-1}\right) q_{J} \geq 0 \quad \text { for all } \quad \zeta \in \mathcal{U} .
$$

Furthermore, $(M(\zeta) z(\zeta)+q)_{N} \geq 0$ for all $\zeta \in \mathcal{U}$ is equivalent to

$$
M_{N, J}(\zeta)\left(\sum_{i \in[k]} \zeta_{i} \tilde{M}^{J, i}-\left(M_{J}^{0}\right)^{-1}\right) q_{J}+q_{N} \geq 0 \quad \text { for all } \quad \zeta \in \mathcal{U} \text {. }
$$


The following theorem summarizes that these conditions lead to a full characterization.

Theorem 6. Let $D$ and $r$ be characterized as in Corollary 4 for $J \subseteq[n]$ such that $M_{0}^{J}$ is invertible. Furthermore, suppose that $D_{[h], .}=0$ holds. Then, $z(\zeta)=D \zeta+r$ is an $A A R$ solution for (12) if and only if $M(\zeta)$ and $q$ fulfill the conditions (14), (16), and (17).

Proof. It only remains to show that $(M(\zeta) z(\zeta)+q)_{J}=0$ for all $\zeta \in \mathcal{U}$ is implied by (14). For all $\zeta \in \mathcal{U}$ we have

$$
\begin{aligned}
(M(\zeta) z(\zeta)+q)_{J} & =M_{J}(\zeta) z_{J}(\zeta)+q_{J} \\
& =M_{J}(\zeta)\left(D_{J,} \cdot \zeta+r_{J}\right)+q_{J} \\
& =M_{J}^{0} D_{J,} \cdot \zeta+\sum_{i=1}^{k} \zeta_{i} M_{J}^{i} D_{J} \cdot \zeta+M_{J}^{0} r_{J}+\sum_{i=1}^{k} \zeta_{i} M_{J}^{i} r_{J}+q_{J} \\
& =M_{J}^{0} D_{J,} \cdot \zeta+\sum_{i=1}^{k} \zeta_{i} M_{J}^{i} D_{J} \cdot \zeta+\sum_{i=1}^{k} \zeta_{i} M_{J}^{i} r_{J}=(*),
\end{aligned}
$$

where we used $M_{J}^{0} r_{J}=-q_{J}$. We apply (15) and obtain

$$
\begin{aligned}
(*) & =M_{J}^{0} \sum_{i=1}^{k} \zeta_{i} \tilde{M}^{J, i} q_{J}+\sum_{i=1}^{k} \zeta_{i} M_{J}^{i} \sum_{j=1}^{k} \zeta_{j} \tilde{M}^{J, j} q_{J}+\sum_{i=1}^{k} \zeta_{i} M_{J}^{i} r_{J} \\
& =\sum_{i=1}^{k} \zeta_{i} M_{J}^{i}\left(M_{J}^{0}\right)^{-1} q_{J}+\sum_{i, j \in[k]} \zeta_{i} \zeta_{j} M_{J}^{i} \tilde{M}^{J, j} q_{J}+\sum_{i=1}^{k} \zeta_{i} M_{J}^{i} r_{J}=(* *) .
\end{aligned}
$$

By (14) we know $\sum_{i, j \in[k]} \zeta_{i} \zeta_{j} M_{J}^{i} \tilde{M}^{J, j} q_{J}=0$. Thus,

$$
\begin{aligned}
(* *) & =\sum_{i=1}^{k} \zeta_{i} M_{J}^{i}\left(M_{J}^{0}\right)^{-1} q_{J}+\sum_{i=1}^{k} \zeta_{i} M_{J}^{i} r_{J} \\
& =\sum_{i=1}^{k} \zeta_{i} M_{J}^{i}\left(M_{J}^{0}\right)^{-1} q_{J}-\sum_{i=1}^{k} \zeta_{i} M_{J}^{i}\left(M_{J}^{0}\right)^{-1} q_{J}=0 .
\end{aligned}
$$

We conclude this section with some final remarks on the derived results and the uniqueness of solutions. Corollary 4 shows that we can fully characterize an AAR solution if the nominal matrix $M_{J}^{0}$ is invertible. In general, the difficulty lies in finding the set $J$ of nonzero entries in the solution. Therefore, there might exist different AAR solutions even if $M_{I}^{0}$ is invertible for every $I \subseteq[n]$. However, if $M^{0}$ is positive definite, $r$ is unique and therefore the set $J$ is unique, yielding the uniqueness of an AAR solution if it exists at all.

Note that we do not state a general existence result here for the case of uncertain $M$ as we did in Corollary 2 for uncertain $q$. We think that an analogous result can be obtained, in principle, by using Theorem 6 and by checking all vertices of the box-uncertainty set for $\zeta$ in (16) and (17). Although finite, the number of conditions in such an existence result most likely would be exponential in the dimension of the uncertainty set. We think that the same also holds for the size of a corresponding mixed-integer programming formulation, which is why we omit to state it here.

Finally, let us also comment on the case in which both the LCP vector $q$ as well as the LCP matrix $M$ are uncertain. The easier setting then is the one in which both uncertainties are independent. However, already this case is rather challenging for affinely adjustable robust LCPs. Consider, for instance, Condition (14), which is 
also part of the final characterization in Theorem 6. A simultaneous consideration of $q$ and $M$ would require that the null-space condition in (14) is satisfied for $q_{J}(u)$ for all $u \in \mathcal{U}_{q}$. Our hypothesis is that this extended condition alone would already be rather hard to satisfy in practically meaningful LCP settings, which is why we postpone the consideration of uncertainty in $q$ and $M$ to future research.

\section{Conclusion}

In this paper, we studied affinely adjustable robust linear complementarity problems with box-uncertainties either in the LCP matrix $M$ or in the LCP vector $q$. We addressed the topics of characterization, existence, and uniqueness of solutions completely for the case of uncertain $q$. Moreover, we developed a mixed-integer linear model that allows to compute affinely adjustable robust LCP solutions with standard solvers. For the case of uncertain $M$, characterizations are established as well and uniqueness of solutions is shown under the assumption that the nominal LCP matrix is positive definite.

While the standard single-stage modeling assumptions of strict as well as of $\Gamma$ robustness both fail to enable the study of robust solutions directly (instead, the LCP's gap function formulation is usually considered), imposing the assumption of affine adjustability in the second stage is sufficient. Thus, adjustable robustness is the first established concept of robust optimization that has been carried over to LCPs, which allows for studying the robust LCP solutions directly instead of considering the gap function formulation as a replacement. However, several problems remain open. For instance, a compact existence result and a compact mixed-integer programming formulation for the case of uncertain LCP matrix is missing. Moreover, the consideration of other uncertainty sets like ellipsoids or the consideration of non-affine decision rules is part of our future research.

\section{ACKNOWLEDGMENTS}

This research has been performed as part of the Energie Campus Nürnberg $(\mathrm{EnCN})$ and is supported by funding of the Bavarian State Government. The authors thank the Deutsche Forschungsgemeinschaft for their support within project A05, B06, and B08 in the Sonderforschungsbereich/Transregio 154 "Mathematical Modelling, Simulation and Optimization using the Example of Gas Networks".

\section{REFERENCES}

[1] I. I. Almaraj and T. B. Trafalis. "Affinely adjustable robust optimization under dynamic uncertainty set for a novel robust closed-loop supply chain." In: Computers \& Industrial Engineering 145 (2020), p. 106521. DOI: 10.1016/j.cie.2020.106521.

[2] D. Aßmann, F. Liers, M. Stingl, and J. Vera. "Deciding Robust Feasibility and Infeasibility Using a Set Containment Approach: An Application to Stationary Passive Gas Network Operations." In: SIAM Journal on Optimization 28 (2018), pp. 2489-2517. DOI: 10.1137/17M112470X.

[3] A. Ben-Tal, L. El Ghaoui, and A. Nemirovski. Robust Optimization. Princeton University Press, 2009.

[4] A. Ben-Tal, A. Goryashko, E. Guslitzer, and A. Nemirovski. "Adjustable robust solutions of uncertain linear programs." In: Mathematical Programming 99.2 (2004), pp. 351-376. DOI: 10.1007/s10107-003-0454-y.

[5] D. Bertsimas, D. B. Brown, and C. Caramanis. "Theory and Applications of Robust Optimization." In: SIAM Review 53.3 (2011), pp. 464-501. DOI: $10.1137 / 080734510$. 
[6] D. Bertsimas and M. Sim. "Robust discrete optimization and network flows." In: Mathematical Programming 98.1 (2003), pp. 49-71. DOI: 10.1007/s10107-003-0396-4.

[7] D. Bertsimas and M. Sim. "The Price of Robustness." In: Operations Research 52.1 (2004), pp. 35-53. DOI: 10.1287/opre.1030.0065.

[8] J. R. Birge and F. Louveaux. Introduction to Stochastic Programming. Springer Science \& Business Media, 2011. DOI: 10.1007/978-1-4614-0237-4.

[9] E. Çelebi, V. Krebs, and M. Schmidt. "Г-Robust Electricity Market Equilibrium Models with Transmission and Generation Investments." In: Energy Systems (2020). DOI: 10.1007/s12667-020-00411-x. Forthcoming.

[10] X. Chen and M. Fukushima. "Expected Residual Minimization Method for Stochastic Linear Complementarity Problems." In: Mathematics of Operations Research 30.4 (2005), pp. 1022-1038. DOI: 10.1287/moor.1050.0160.

[11] X. Chen, R. J.-B. Wets, and Y. Zhang. "Stochastic Variational Inequalities: Residual Minimization Smoothing Sample Average Approximations." In: SIAM Journal on Optimization 22.2 (2012), pp. 649-673. DOI: $10.1137 / 110825248$.

[12] X. Chen, C. Zhang, and M. Fukushima. "Robust solution of monotone stochastic linear complementarity problems." In: Mathematical Programming 117.1 (2009), pp. 51-80. DOI: 10.1007/s10107-007-0163-z.

[13] R. W. Cottle, J.-S. Pang, and R. E. Stone. The Linear Complementarity Problem. Society for Industrial and Applied Mathematics, 2009. DOI: 10.1137/1.9780898719000.

[14] M. Fischetti and M. Monaci. "Light Robustness." In: Robust and Online Large-Scale Optimization: Models and Techniques for Transportation Systems. Ed. by R. K. Ahuja, R. H. Möhring, and C. D. Zaroliagis. Berlin, Heidelberg: Springer Berlin Heidelberg, 2009, pp. 61-84. DOI: 10.1007/978-3-642-05465-5_3.

[15] S. A. Gabriel, A. J. Conejo, J. D. Fuller, B. F. Hobbs, and C. Ruiz. Complementarity modeling in energy markets. Vol. 180. Springer Science \& Business Media, 2012.

[16] B. F. Hobbs. "LCP models of Nash-Cournot competition in bilateral and POOLCO-based power markets." In: IEEE Power Engineering Society. 1999 Winter Meeting. Vol. 1. 1999, pp. 303-308. DOI: 10.1109/PESW.1999.747471.

[17] B. F. Hobbs and U. Helman. "Complementarity-Based Equilibrium Modeling for Electric Power Markets." In: Modeling Prices in Competitive Electricity Markets. Ed. by D. Bunn. London: Wiley, 2004.

[18] P. Kall and S. W. Wallace. Stochastic Programming. Wiley-Interscience Series in Systems and Optimization. New York: Wiley, 1994.

[19] T. Kleinert and M. Schmidt. "Global Optimization of Multilevel Electricity Market Models Including Network Design and Graph Partitioning." In: Discrete Optimization 33 (2019), pp. 43-69. DOI: 10.1016/j.disopt.2019.02.002.

[20] A. Kramer, V. Krebs, and M. Schmidt. Strictly and $\Gamma$-Robust Counterparts of Electricity Market Models: Perfect Competition and Nash-Cournot Equilibria. Tech. rep. 2018. URL: http://www.optimization-online.org/DB_HTML/2018/07/6709.html.

[21] V. Krebs, M. Müller, and M. Schmidt. "Г-Robust Linear Complementarity Problems with Ellipsoidal Uncertainty Sets." In: International Transactions in Operational Research (2021). DOI: 10.1111/itor.12988. Forthcoming.

[22] V. Krebs and M. Schmidt. " $\Gamma$-Robust Linear Complementarity Problems." In: Optimization Methods and Software (2020). DOI: 10.1080/10556788.2020.1825708. Forthcoming. 
[23] G.-H. Lin and M. Fukushima. "New reformulations for stochastic nonlinear complementarity problems." In: Optimization Methods and Software 21.4 (2006), pp. 551-564. DOI: 10.1080/10556780600627610.

[24] J. Mather and E. Munsing. "Robust Cournot-Bertrand equilibria on power networks." In: American Control Conference (ACC), 2017. IEEE. 2017, pp. 2747-2754. DOI: 10.23919/ACC. 2017.7963367.

[25] C. B. Metzler, B. F. Hobbs, and J.-S. Pang. "Nash-Cournot Equilibria in Power Markets on a Linearized DC Network with Arbitrage: Formulations and Properties." In: Networks and Spatial Economics 3.2 (2003), pp. 123-150. DOI: $10.1023 / \mathrm{A}: 1023907818360$.

[26] A. Ouorou. "Affine Decision Rules for Tractable Approximations to Robust Capacity Planning in Telecommunications." In: Network Optimization. Ed. by J. Pahl, T. Reiners, and S. Voß. Berlin, Heidelberg: Springer Berlin Heidelberg, 2011, pp. 277-282. DOI: 10.1007/978-3-642-21527-8_32.

[27] P. M. Pardalos. "Linear complementarity problems solvable by integer programming." In: Optimization 19.4 (1988), pp. 467-474. DOI: 10.1080/02331938808843365.

[28] M. Poss and C. Raack. "Affine Recourse for the Robust Network Design Problem: Between Static and Dynamic Routing." In: Network Optimization. Ed. by J. Pahl, T. Reiners, and S. Voß. Berlin, Heidelberg: Springer Berlin Heidelberg, 2011, pp. 150-155. DOI: 10.1007/978-3-642-21527-8_19.

[29] M. Sim. "Robust Optimization." PhD thesis. Massachusetts Institute of Technology, 2004.

[30] A. L. Soyster. "Technical Note Convex Programming with Set-Inclusive Constraints and Applications to Inexact Linear Programming." In: Operations Research 21.5 (1973), pp. 1154-1157. DOI: 10.1287/opre.21.5.1154.

[31] D. Wu, J.-Y. Han, and J.-H. Zhu. "Robust solutions to uncertain linear complementarity problems." In: Acta Mathematicae Applicatae Sinica, English Series 27.2 (2011), pp. 339-352. DOI: 10.1007/s10255-010-0033-y.

[32] Y. Xie and U. V. Shanbhag. "On Robust Solutions to Uncertain Linear Complementarity Problems and their Variants." In: SIAM Journal on Optimization 26.4 (2016), pp. 2120-2159. DOI: 10.1137/15M1010427.

[33] Y. Xie and U. V. Shanbhag. "On robust solutions to uncertain monotone linear complementarity problems (LCPs) and their variants." In: 2014 IEEE 53rd Annual Conference on Decision and Control (CDC). IEEE. 2014, pp. 28342839. DOI: 10.1109/CDC . 2014.7039824.

[34] İ. Yanıkoğlu, B. Gorissen, and D. den Hertog. "A Survey of Adjustable Robust Optimization." In: European Journal of Operational Research 277 (2019), pp. 799-813. DOI: $10.1016 /$ j.ejor.2018.08.031.

(C. Biefel, F. Liers, J. Rolfes) Friedrich-Alexander-Universität Erlangen-Nürnberg, Discrete Optimization, Cauerstr. 11, 91058 Erlangen, Germany; Energie Campus Nürnberg, Fürther Str. 250, 90429 Nürnberg, Germany

Email address: \{christian.biefel,frauke.liers,jan.rolfes\}@fau.de

(M. Schmidt) Trier University, Department of Mathematics, Universitätsring 15, 54296 Trier, Germany

Email address: martin.schmidt@uni-trier.de 